\title{
LAPARASCOPIC-ASSISTED ENDOSCOPIC MUCOSAL RESECTION OF A POLYP IN SIGMA AND LAPAROSCOPIC RIGHT HEMICOLECTOMY FOR ADENOCARCINOMA IN COLON ASCENDENS - CASE REPORT
}

\author{
Ivan Lyutakov ${ }^{1}$, Plamen Penchev ${ }^{1}$, Branimir Golemanov , Georgi Jelev², Emil Kostadinov², \\ Ivan Terziev ${ }^{3}$, Borislav Vladimirov ${ }^{1}$ \\ ${ }^{1}$ Clinic of Gastroenterology, University Hospital “Tsaritsa Yoanna - ISUL”, Sofia, Bulgaria \\ ${ }^{2}$ Clinic of Surgery, University Hospital "Tsaritsa Yoanna - ISUL”, Sofia, Bulgaria \\ ${ }^{3}$ Department of Pathology, University Hospital “Tsaritsa Yoanna - ISUL”, Sofia, Bulgaria
}

\begin{abstract}
Today, endoscopic polypectomy has become a daily-performed routine procedure in the operating room. Almost every polyp in the colon can be removed endoscopically, but there are some problematic adenomas which are either large in size or difficult to approach. We present 80 years old white woman with a tubulovillous adenoma with high-grade dysplasia on a flat surface located in sigma with $4 \times 5 \mathrm{~cm}$ in size and also synchronously found in colon ascendens a well differentiated G1 adenocarcinoma with $2 \times 2 \mathrm{~cm}$ in size on a flat surface. The abdominal ultrasound did not find any liver metastases. Because of the difficult location and the size of the two lesions in the right and left colon, we make a decision for a laparoscopic-assisted endoscopic mucosal resection (piece-meal) of the polyp in sigma and for the adenocarcinoma in colon ascendens the procedure was followed by laparoscopic right hemicolectomy in one step approach. The both interventions were performed without complications and good postoperative period. Hybrid endoscopic and laparoscopic interventions are very effective approach that can resect large tubulovillous polyps and adenocarcinoma of the colon, with a lesser risk of complications. Combining the methods in one surgery save time, make endoscopy more easy to perform and can save one-half of the colon in cases of synchronous lesions of the colon. Scr Sci Med 2017; 49(3): 49-52
\end{abstract}

Keywords: Endoscopic mucosal resection, Laparoscopic surgery, Polypectomy, Tubulovillous adenoma, Colon adenocarcinoma

\footnotetext{
Address for correspondence:

Ivan Lyutakov

Clinic of Gastroenterology,

University Hospital "Tsaritsa Yoanna - ISUL"

Sofia, Bulgaria

8 Byalo More St, 1527 Sofia

e-mail:ivan.lutakov@gmail.com
}

Received: July 27, 2017

Accepted: September 15, 2017

\section{INTRODUCTION}

Primary prevention of colorectal cancer depends on effective screening through colonoscopy and polypectomy (1). Complete resection of some polyps can be technical challenges particularly when polyps are flat, large, or behind colonic folds. Laparoscopy is a helpful method to the endoscopic arsenal in removing difficult colonic polyps without subjecting patients to radical segmental colectomy (2) and was deemed unresectable based on size, broad base, or location of the polyp.

\section{MAIN OUTCOME MEASURES}

Intraoperative and postoperative complications, length of hospital stay, and recurrence were the 
primary outcomes measured. RESULTS Thirty consecutive patients underwent combined endoscopiclaparoscopic surgery. In the last two decades laparoscopically assisted colonoscopic polypectomy (or also known as hybrid laparoendoscopic techniques) has become established in the endoscopic field, which reduced the need of operations for those patients with lesions that are large or hard to approach. These techniques are reported in literature as alternatives to segmental or large colectomy for the treatment of so called "difficult polyps".

The first laparoscopically monitored colonoscopic polypectomy was performed by Franklin in 1991 (3) and reported in 1993 (4). Laparoscopic-assisted colonoscopic polypectomy is the most often used technique and also has the power of laparoscopy to aid endoscopic polypectomy by flattening folds, mobilizing flexures and also providing retraction (2) and was deemed unresectable based on size, broad base, or location of the polyp.

There are several reviews on how to manage the "difficult polyp" endoscopically $(5,6)$. The use of hybrid techniques in dealing with "difficult polyps" is usually a last resort maneuver when all other options are exhausted (1). At the present time with the advance of the endoscopic equipment, endoscopic training and mastering the techniques such as endoscopic mucosal resection (EMR) and endoscopic submucosal dissection (ESD), most of the polyps can be resected and very few of them will require the assistance of laparoscopy. The mortality associated with laparoscopic colon resection is about $1 \%$ and overall morbidity about $18 \%$ (7)less morbidity, improved reconvalescence and better quality of life. The assistance of laparoscopy will definitively be helpful at some polyps or lesions that are suspicious for adenocarcinoma.

We present a case report of a patient who have been diagnosed with two different lesions located in the sigma and colon ascendens and underwent laparoscopically assisted polypectomy followed by laparoscopy right hemicolectomy in one step approach.

The aim of this case report is to show that synchronously found two lesions (giant adenoma of the sigma and adenocarcinoma of the colon ascendens) can be removed using minimally invasive techniques in one step approach and save one-half of the colon of the patient.

\section{CASE REPORT}

We present 80 years old white woman, with performed ambulatory a screening colonoscopy that found a sessile tubulovillous adenoma with highgrade dysplasia on a flat surface located in sigma with $4 \times 5 \mathrm{~cm}$ in size and also synchronously in colon ascendens a well differentiated G1 adenocarcinoma with $2 \times 2 \mathrm{~cm}$ in size on a flat surface. The abdominal ultrasound did not find any liver metastases. Because of the difficult location and the size of the two lesions in the right and left colon, we have decided to performe laparoscopic-assisted EMR (piece-meal) of the polyp in sigma and for the adenocarcinoma in colon ascendens the procedure has been done a laparoscopic right hemicolectomy in one step approach.

The patient was admitted to the hospital and during this hospitalization, the patient underwent the mentioned procedure into the operating room. The operation was performed under general anesthesia. The patient was in a supine position during the operation. Laparoscopy was performed using an Olympus laparoscope. Pneumoperitoneum was formed by using $\mathrm{CO}_{2}$ gas. During laparoscopy, abdominal pressure was maintained around $10 \mathrm{~mm}$ $\mathrm{Hg}$. All actions and procedures in the peritoneal cavity were performed using soft intestinal graspers. After that, we started with colonoscopy performed using an Olympus EVIS EXERA III F-HQ190L/I colonoscope with the diathermal snare for "piece-meal" EMR of the adenoma in the left colon. A colonoscopic injection needle was used to inject saline and methylene blue for lifting the lesion. Submucosal injection beneath the flat mucosal lesion lifts it away from the muscle layer and it became more easy to resect. In our case, the lesion in the sigma was with good "lifting sign". We have tried to remove the whole polyp at a single sitting but in cases like this, when the lesions is $>2 \mathrm{~cm}$, we have considered a piecemeal resection. Sometimes, haustral folds can create blind spots for the endoscopist, making excision of the entire polyp challenging and increasing the risk of bowel wall injury or perforation. Also, the polyp was behind a haustral fold and the laparoscopic instruments were used to present the polyp to the colonoscope. We have performed this procedure by using 
gentle pressure on the colon wall with a laparoscopic grasper to flatten out the haustral fold. The idea of this move is to improve the endoscopic view upon the lesion and stabilization of the polyp for the duration of its resection. This has to be assisted by adjustments of the laparoscopic instruments as needed to help EMR (Fig. 1).

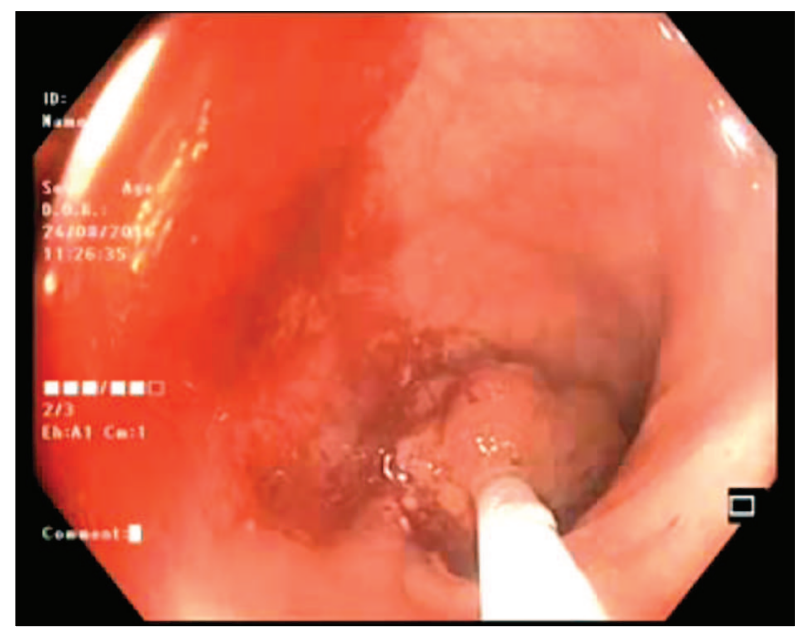

Figure 1. Starting the piece-meal resection of the lesion in sigma

For better comfort and safety of the manipulations, we suggest having laparoscopic access and instruments in place before inserting the colonoscope and distending the bowel. Because of the size of the polyp of the sigma we have proceeded to piece-meal resection and resecting submucosal layer with continuously re-injecting saline and methylene blue for lifting the lesion. After resection was performed, an inspection with Narrow-band imaging (NBI) and "Near focus" has been used for a residual adenomatous tissue. Remaining parts of the adenoma has been destroyed using argon-plasma coagulation (APC) and the margins of the resected site were ablated by APC. The resection site has been cleaned and checked for bleeding and perforation possibility. After finishing EMR we have reached the second lesion into the colon ascendens (that was previously diagnosed by histology) that was adenocarcinoma G1 with size $2 \times 2 \mathrm{~cm}$ (Fig. 2).

The lesion has been inspected using NBI and amorphous or absent surface pattern classified as NICE type 3 classifications is shown in Figure 3.

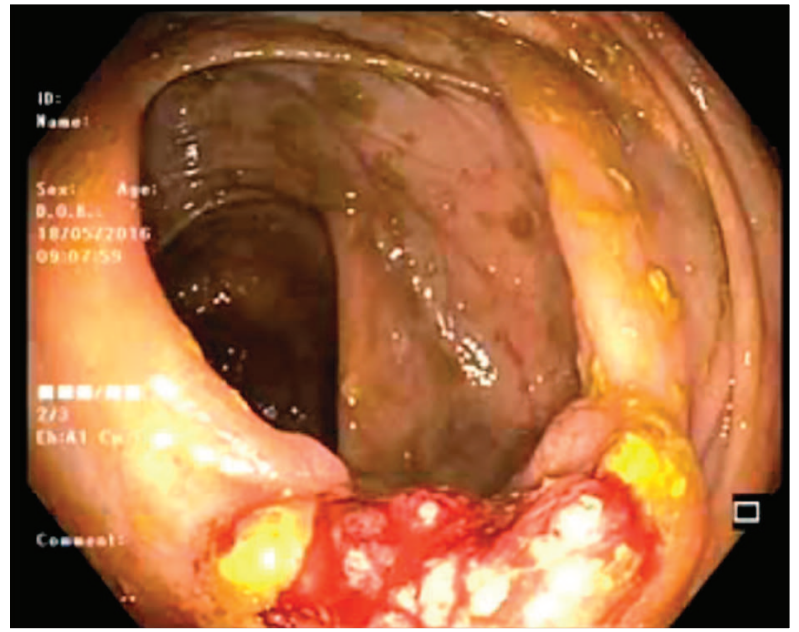

FIgure 2. The endoscopic view of the lesion that is in colon ascendens

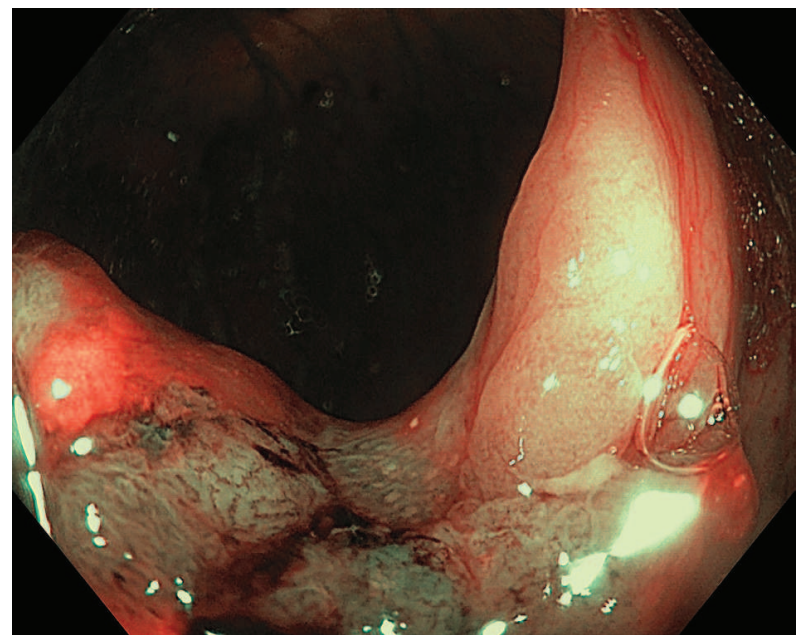

Figure 3. Using narrow-band imaging for the lesion in the colon ascendens: Amorphous or absent surface pattern classified as NICE type 3 classifications

After the EMR was finished, a laparoscopicassisted right hemicolectomy with transverse right paraumbilical minilaparotomy has been performed for the lesion in the colon ascendens. Starting with the mobilization of the terminal ileum, the right lateral peritoneal reflection is opened, the right ureter is identified and also protected. The cecum and ascending colon are mobilized medially. Firstly, the hepatic flexure and gastrocolic ligament have been divided to the level of the mid transverse colon and once the colon is freely mobilized to the midline, the terminal ileum and right colon are exteriorized by extending the supraumbilical incision to a length of 4 to $6 \mathrm{~cm}$. Secondly, the mesentery and bowel have 
Laparascopic-Assisted Endoscopic Mucosal Resection of a Polyp in Sigma and Laparoscopic Right Hemicolectomy for Adenocarcinoma ...

been divided, then the anastomosis is constructed, the bowel is returned to the peritoneal cavity, and the incisions are closed. The estimated length of the both procedures is 105 minutes and hospital stay was 4 days. The both interventions were performed without complications and good postoperative period. It was found a T2N0M0 adenocarcinoma G1 in histology evaluation for the lesion in the right colon and this was not suitable for ESD. The second lesion into the sigma was a tubulovillous adenoma with high-grade dysplasia. The patient has been followed-up.

\section{DISCUSSION}

Laparoscopic-assisted colonoscopic polypectomy sparing patients the morbidity associated with major bowel resection (8). This hybrid technique appears to be the one used most frequently when a combined laparoendoscopic approach is required for removing a difficult polyp or lesions that are suspicious for adenocarcinoma. The description of the technique is quite uniform with the exception of a few details. Most groups have used CO2 insufflation for endoscopy to facilitate visualization during laparoscopy (9), but the procedure can also be successfully performed with air insufflation. Presenting the polyp to the endoscopist using laparoscopic instruments is the hallmark of this method (10). Nowadays the endoscopic removal of benign colorectal lesions is the gold standard, but there are some polyps that are hard to remove because of their size or their inaccessible growth place and when it combined with a synchronous lesion it is very appropriate to use these hybrid techniques. The role and benefit of this hybrid technique is continually evolving and may depend on the local endoscopic expertise available.

\section{CONCLUSION}

Hybrid endoscopic and laparoscopic interventions is useful approach for resecting large tubulovillous polyps and synchronous adenocarcinoma of the colon, with a lesser risk of complications. Combining the methods in one surgery save time, make endoscopy more easy to perform and can save one-half of the colon in cases of synchronous lesions of the colon

\section{REFERENCES}

1. Aslani N, Alkhamesi NA, Schlachta CM. Hybrid Laparoendoscopic approaches to endoscopi- cally unresectable colon polyps. J Laparoendosc Adv Surg Tech A. 2016;26(8):581-90. doi: 10.1089/ lap.2015.0290.

2. Crawford AB, Yang I, Wu RC, Moloo H, Boushey RP. Dynamic article: combined endoscopic-laparoscopic surgery for complex colonic polyps: postoperative outcomes and video demonstration of 3 key operative techniques. Dis Colon Rectum. 2015;58(3):363-9. doi: 10.1097/ DCR.0000000000000311.

3. Prohm P, Weber J, Bönner C. Laparoscopic-assisted coloscopic polypectomy. Dis Colon Rectum. 2001;44(5):746-748.

4. Anonymous. SAGES Meeting. SAGES Meet March 31-April 3. 1993; Phoenix, A.

5. Zhang M, Shin EJ. Successful endoscopic strategies for difficult polypectomy. Curr Opin Gastroenterol. 2013 Sep;29(5):489-894. doi: 10.1097/ MOG.0b013e3283646e89.

6. Pishvaian AC, Al-Kawas FH. Retroflexion in the colon: a useful and safe technique in the evaluation and resection of sessile polyps during colonoscopy. Am J Gastroenterol. 2006;101(7):147983. doi: 10.1111/j.1572-0241.2006.00606.x.

7. Schwenk W, Haase O, Neudecker JJ, Müller JM. Short term benefits for laparoscopic colorectal resection. In: Schwenk W, editor. Cochrane Database of Systematic Reviews. Chichester, UK: John Wiley \& Sons, Ltd; 2005. p. CD003145.

8. Beck DE, Karulf RE. Laparoscopic-assisted fullthickness endoscopic polypectomy. Dis Colon Rectum. 1993;36(7):693-5.

9. Goh C, Burke JP, McNamara DA, Cahill RA, Deasy J. Endolaparoscopic removal of colonic polyps. Colorectal Dis. 2014;16(4):271-5. doi: 10.1111/codi.12512.

10. Mikalauskas $S$, Račkauskas $R$, Zeromskas $P$, Strupas K. Giant Bauhin valve adenoma and laparoscopically assisted colonoscopic polypectomy. Wideochir Inne Tech Maloinwazyjne. 2014;9(3):484-5. doi: 10.5114/wiitm.2014.45045. 